\title{
The Importance of Longitudinal Studies in Family Medicine: Experiences of Two Practice-based Research Networks
}

\author{
Chris van Weel, MD, PhD, FRCGP, Evelyn van Weel-Baumgarten, $M D, P h D$, and \\ Jim Mold, MD, $M P H$
}

Background: For evidence-based decision making in family practice, it is essential to know the longterm (natural) course of common diseases and their outcomes under care and treatment. This article, based on a research methodology workshop, aims to raise awareness and interest in longitudinal research in practice-based research networks (PBRNs) among family physicians (FP) and researchers, and to elucidate a number of broad principles that apply to longitudinal research in such settings.

Methods: A workshop discussion of PBRN experts, based on 2 examples of PBRN-based longitudinal studies, focusing on the general methodological principles was held in March 2005 in Colorado Springs, Co. The first study, conducted in a historic cohort, documented long-term outcome of depression in 386 patients in family practice. The second study analyzed consequences of age-associated peripheral neuropathy in a concurrent follow-up study design. It was possible to prospectively follow a cohort of 604 healthy subjects enrolled in the practices by research nurses. Discussion at the workshop was generated around the following methodological topics: study designs, recruitment, retention and tracking of participants, dealing with changes in diagnostic and treatment of standards over time, informed consent, data analysis, and funding issues.

Results and Conclusions: The workshop concluded that, although there are many obstacles to be overcome when conducting longitudinal studies in practice settings, the benefits for our discipline could be substantial. Concurrent and historic cohorts each have advantages and disadvantages. Although tracking patients (and physicians) over time is a challenge, periodic rewards enhance recruitment and retention. Informed consent is critical although ethical requirements may change. Use of standard welldefined definitions promotes consistency of data collection over time, as does training of FPs and staff. Data analysis has become both easier and more sophisticated, and statistical assistance is advised from the outset. Funding remains a challenge, but costs need not be prohibitive. Given its importance, PBRNs should be encouraged to develop methods of long-term data collection on cohorts of patients followed by primary care physicians. (J Am Board Fam Med 2006;19:69-74.)

Continuity of care is a hallmark of family medicine. ${ }^{1,2}$ To promote evidence-based decision mak-

Submitted 27 May 2005; revised 12 September 2005; accepted 16 September 2005.

From the Department of Family Medicine, Radboud University Medical Center Nijmegen, Nijmegen, The Netherlands (CvW, EvW-B); and Department of Family and Preventive Medicine, University of Oklahoma Health Sciences Center, Oklahoma City, OK (JWM).

Based on a workshop at the annual conference of the Federation of Practice-based Research Networks, Colorado Springs, CO, 3-7 March 2005.

Conflict of interest: none declared.

Corresponding author: James W. Mold, MD, MPH, Department of Family and Preventive Medicine, University of Oklahoma Health Sciences Center, 900 NE 10th Street, Oklahoma City, OK 73104 (E-mail: james-mold@ouhsc.edu). ing, it is therefore essential to have information that encompasses the natural course of common diseases, the long-term outcomes of care and treatment, and the impact of primary care interventions on these outcomes. There is currently a paucity of longitudinal research conducted in primary care settings on which to base clinical decisions. ${ }^{3}$ This hampers the relevance of research for primary care practice. Family physicians (FP) make a core contribution to the health status of patients in the community and of crucial interest are the lasting effects of their interventions-from watchful waiting, advice and patient-empowerment, to early diagnosis and treatment. Long-term studies can support FPs in making the best decisions in treating 
their patients. Because primary care practice-based research networks (PBRNs) rely heavily on relationships between individual practices and their patients across time, they ought to provide ideal environments for longitudinal research.

However, awareness of the possibility and clinical relevance of longitudinal studies are not generally acknowledged-not even in family medicine. ${ }^{3}$ This article describes a workshop at which 2 examples of completed longitudinal studies were used to generate discussion about methodological issues related to conducting longitudinal studies in PBRNs. The primary objectives are to raise awareness and interest in longitudinal research in PBRNs among FPs and researchers, and to elucidate a number of broad principles that apply to longitudinal research in such settings.

\section{Methods}

This article is based on a workshop of PBRN experts held at the 2005 Annual Convocation of Practice-Based Research Networks in Kansas City. First, it summarizes 2 PBRN-based longitudinal studies that served in the workshop as examples of success, with emphasis on methodology of longitudinal research, with references guiding readers interested in the details of these studies to the available publications. The examples were used as a springboard for discussing general methodological principles and challenges associated with conducting longitudinal studies in PBRNs.

The choice of the 2 examples was, by and large, for convenience: the workshop presenters had developed their personal experience in the context of these studies, and they were able to answer questions of workshop participants with reference to project-specific empirical information. Otherwise the choice of the 2 examples was nonsignificant.

\section{Example 1: Long-Term Outcomes Associated with Depression in Family Practice}

Background: Depression is a common chronic condition, for which long-term treatment with antidepressive medication is often recommended to prevent recurrent episodes. This is based primarily on research from secondary care psychiatric settings.

Objective: To study the long-term outcome of depression in primary care settings.

Design: A nonconcurrent (historical or retrospective) cohort analysis was performed using all patients from the Nijmegen Family Medicine database $^{3}$ with an episode of depression who could be followed-up for at least 10 years after their first episode. ${ }^{4}$ To validate the diagnosis of depression a cohort of patients from the research practices with a new or existing code of depression at the time of study (1997) underwent a standardized psychiatric interview (Composite International Diagnostic Interview, CIDI).

Setting: The Nijmegen academic PBRN, The Netherlands. ${ }^{5}$ The PBRN records since 1971, which contained all new episodes of morbidity presented to the FP, including diagnoses made in secondary care after referral of the patient (Nijmegen Continuous Morbidity Recording). ${ }^{3-5}$

Participants: All patients with a family physician diagnosed episode of depression during the years 1971 to 1984 and in1997.

Outcome measures: Recurrence of depression after first episode, referrals, and suicide (attempts) were retrieved from the morbidity records. Quality of life was measured with the Rand-36, in a mailed questionnaire.

Results: There were 386 patients with a first recorded episode of depression between 1971 and 1984, who were considered for the study. Of this group, $107 \mathrm{had}$ left the practice before the time of study in 1995 and 46 had died. Because of administrative errors 11 had to be excluded, leaving 222 with complete follow-up from their (first) depression episode for 10 years or longer. In this group, $60 \%$ did not have a recurrent episode, in $15 \%$, a referral to psychiatric care took place and $9 \%$ were admitted to a psychiatric care unit. There were 10 suicide attempts ( 8 patients). There were 2 cases of suicide in $6382+$ observation years, which did not differ significantly from the 13 suicides in nondepressed patients in the database with 285,008 observation years. ${ }^{4}$

The validation psychiatric interview was conducted in 99 patients: all patients with an FPdiagnosed depression (45) in the year preceding the study in 1997 were invited for this study of whom 33 consented. For each case 2 sex-age and FP/ practice-matched controls were selected: one with chronic nervous-functional complaints and one without a mental health diagnosis. ${ }^{6}$ It confirmed the specificity and sensitivity of the FP diagnosis: of the 33 cases with an FP diagnosis of depression 28 had had a depression confirmed according to the criteria of the Diagnostic and Statistical Manual of 
mental disorders- $4^{\text {th }}$ edition (DSM-IV), 7 of the patients with chronic nervous-functional complaints, and none of those without an FP diagnosed mental health problem. ${ }^{6}$ It also made clear that the history of depression had had a major impact on the patient's quality of life. ${ }^{7}$

Conclusions: Family physicians, in general, diagnose depression according to the DSM-IV criteria. Depression in family practice is restricted to a single episode in a majority of cases. Nevertheless, for a substantial minority, the impact is substantial and lasting. Suicides are relatively rare in this population of patients.

\section{Example 2: Consequences of Age-Associated Peripheral Neuropathy; “Oklahoma Studies"}

Background: Age-associated peripheral neuropathy has been considered to be a normal finding of little importance to health. However, cross-sectional data have suggested that it may not be completely benign. ${ }^{8}$

Objective: To determine whether age-associated peripheral neuropathy (AAPN) is associated with adverse health events.

Design: Longitudinal study involving a cohort of patients 65 years of age and older drawn from the practices of primary care physicians in a PBRN in Oklahoma, and followed annually for up to 5 years. Patients with a history of diseases known to cause neuropathy were excluded. Survival analyses were performed to analyze the association between AAPN and events.

Setting: The Oklahoma Physicians Resource/ Research Network.

Participants: 604 subjects with no history of autoimmune disorders, B12 deficiency, diabetes, chronic hepatitis, chronic renal failure, Crohn disease, hereditary neuropathy, or sarcoidosis.

Independent variables: Peripheral neuropathy was defined as bilateral absence of one or more of the following: ankle reflex, sense of touch (feet), position sensation (great toe), and vibration sensation (ankle) as determined by physical examination.

Outcome measures: Death, first hospitalization, first emergency department (ED) visit, first fall, health-related quality of life (Quality of Well-Being-Self Administered and Health Utility Index-3), physical function (Medical Outcomes Study Short Form-36), bodily pain (Medical Outcomes Study Short Form-36), and self-rated health.
Results: After controlling for socio-demographic and disease-related variables, the presence of AAPN predicted time to first hospitalization $(P=$ $.03)$ and death $(P=.0007)$. It was not associated with time to first ED visit or time to first fall.

Conclusions: Age-associated peripheral neuropathy may be a significant predictor of subsequent mortality and morbidity although the reasons are not yet clear.

\section{Results and Conclusions}

The 2 examples referred to in this paper illustrate the possibility of studying, in PBRN settings, patients over extended periods of time and in this way develop research evidence of clinical interventions directed at patients' long-term health status. Both studies were based on representative samples of primary care patients, and it is reasonable to assume their findings hold for other primary care patient populations. But where FP performance would become part of the study, the academic setting of the PBRN of the depression study would hamper generalization. ${ }^{3}$ Within the context of these 2 examples, the following issues of longitudinal research were discussed by the approximately 20 PBRN researchers attending the workshop.

\section{Design Options}

Longitudinal studies can be either concurrent (enrolling and then following patients forward in time) as in the second example, or nonconcurrent (reaching back in time to find a cohort and then identifying outcomes as they subsequently occurred) as in the first example. This terminology is preferred to "prospective and retrospective cohort studies" to avoid confusing retrospective cohort studies with case-control studies, which are almost always retrospective.

The generally accepted theoretical advantages of a concurrent design are the opportunity for more specific and consistent data collection at the onset, and closer follow-up and tracking of outcomes as they occur. The advantage of nonconcurrent designs is the ability to more efficiently collect and analyze data collected over longer time frames.

\section{Recruitment and Retention of Subjects}

The most critical aspect of longitudinal research is the ability to track patients and their outcomes over extended periods of time. This is not a trivial mat- 
ter. Several options available to PBRNs are; (1) to capitalize on the stability of some patient populations and their on-going listing with their personal FP, as in the first example; or (2) to assemble a cohort of patients willing to be followed over time, as in the second example.

Stable populations are, to some extent, the best choice when developing a longitudinal database. In fact, the Nijmegen database was originally designed, in 1967 to 1971 , not specifically for research purposes, but, because of the standardization of data collection and the stability of the population, it has been possible to conduct a variety of longitudinal studies involving this population. ${ }^{3}$ There is a possibility - or even likelihood -that cohorts that were initially established for a specific research question are to some degree biased by the enrolment process, and part of the methodology in building a longitudinal study on such a cohort is an explicit review of bias -and a critical review if its implications for the study.

Rural communities in the United States might be more stable, in general, than urban ones. Even in stable populations, however, people often move. In both PBRNs, efforts are made to keep track of patients who have left the practices, which enables follow-up beyond the period patients receive care through the practice. It is only realistic, though, to acknowledge that without the personal FP-patient relationship, participation in such follow-up is likely to be lower.

In the Oklahoma studies cohort, patients were recruited from lists derived from the billing systems of participating practices. Letters of invitation were then sent out by the physicians themselves, followed by a phone call from the study coordinator. Patients were excluded if they were living in a nursing home, had switched primary care physicians, or were too confused to give informed consent. Patients were re-enrolled each year, which, in retrospect, was a bad idea. Retention would likely have been better if they had been asked to make a 5 -year commitment. Only 242 of the original 604 participants completed all 5 years of data collection.

However, although only patients are recruited to the study, both physicians and patients should be rewarded for continued involvement with feedback about study findings, acknowledgment of appreciation of their role in the study, coverage of study-related costs and, for physicians, ownership as (co)-researcher and (co)author when appropri- ate. In addition, every feasible piece of identifying information that can be ethically obtained from participants should be obtained at the time of enrolment. This should include, when possible, date of birth, race and ethnicity, social security number, driver's license number, employer (including address and phone number), spouse's employer (including address and phone number), and the name, address, and phone number of one or more people who will know the participant's whereabouts.

\section{Dealing with Changing Practice Routines over Time}

Not only do patients and FPs change over time, but also the registration and recording of data in regular practice. During a period of the first study, the participating practices moved from pencil and paper recording of patient files, to computerization of practices. In electronic records the role of coding and classification for routine care is much more relevant than in the era of the "paper office." In the Dutch family practice, the College's guidelines for care have been integrated into the patients' files, through the International Classification of Primary Care (ICPC) coding.

In addition, definitions of diseases may change (the DSM-IV, for example, was introduced in the 1990-s, in the middle of the observation period of the first study example), and new tests and technology will be introduced, which directly influences the care patients receive. Essential in longitudinal research, though, is that data continue to mean the same thing over time, as much as possible. For that reason there is a need to substantiate the value of data recorded in the past (eg, diagnoses in the depression study). This may be second-best to prospective research-specific recording but it makes it possible to address the question of data drift from an empirical basis over time in an open and explicit way.

To enhance the consistency of data, a number of measures can be taken at the time of data collection:

- Use of standardized criteria and definitions and the training of FPs and their staff in their application.

- Continued use over time of the same methodology. In the Nijmegen PBRN the morbidity classification that was used in 1971 , is still used today, to guarantee comparability.

- When definitions change, or new criteria are applied, the essential difference and its date of 
introduction should be recorded. This at least makes it possible to explain the (in)comparability of data over time.

- When an important outcome is death, information can often be obtained or verified using existing death registries even when participants are lost to follow-up.

\section{Informed Consent}

Longitudinal research asks for the ongoing commitment of patients and this raises an ethical question of informed consent. Participants should generally be consented for the expected duration of the study. However, the concept of informed consent is dynamic, and changes over time. This can interfere with longitudinal studies, as it is unclear whether new concepts should be applied for future data collection or also backwards to information already gathered.

The most striking example here is the Nijmegen database that started collecting patients' data at a time in the 1970s, when informed consent was not detailed, at all, in biomedical research. Although informed consent was introduced to all patients on the practice list nearly 20 years ago, a substantial number of data were collected before that time. In the Oklahoma study, participants were re-consented each year. This may have improved recruitment whereas, perhaps appropriately, increasing the subsequent drop-out rate.

Participants in the workshop had a variety of opinions about what such a consent process might need to include. For example, in Olmstead County, Minnesota, involvement in longitudinal studies has become a standard expectation and a source of community pride. Patients sign a generic consent form when they enroll with a physician in the county, and very few refuse.

\section{Data Analysis}

Although excellent methods now exist for analyzing longitudinal data, these analyses are almost always complex enough to require the assistance of a biostatistician. In general, the outcomes of greatest interest in longitudinal analyses are either events (eg, death, hospitalization) or measures of quality of life (eg, functional status, pain). Survival analysis techniques apply equally well to any event (eg, first hospitalization, first emergency department visit) that may occur at some point in the future. A time variable is included so that subjects are only counted during the time they were in the study and at risk for the event.

Mixed regression models may be used to analyze data when the outcome variable is continuous (eg, quality of life). In this case, all measurements available on each subject can be taken into account, giving somewhat more weight to the results from persons with more measurements. Analysis of more than 2 identical measurements in patients over time warrants "repeated measurements" techniques about which entire books have been written. ${ }^{9-12}$ When participants have been drawn from more than one practice and/or when more than one clinician is involved in each practice, hierarchical modeling techniques may be required. ${ }^{13-16}$ Issues involving management of missing data can also be challenging yet important.

\section{Funding Longitudinal Research}

Funding has been one of the biggest challenges for primary care researchers. Longitudinal studies are generally more difficult to fund than short-term projects. Fortunately, studies don't have to be prohibitively expensive, and these expenses can often be distributed over long periods of time. Obligatory costs include initial recruitment and enrolment of patients, training of data collectors, tracking, follow-up, and retention of participants, and data management and analysis. Some of these activities can be considered investments and can be covered by academic departments.

The first project required no external funding at all until the data analysis phase. It did, of course, require some small amounts of clinician time over many years to capture and record the necessary data. The data now support the training of primary care researchers. Applications for funding are then project-specific. The Oklahoma project was funded by a local foundation affiliated with an academic medical center. The foundation believed that an investment in the establishment of the cohort would lead to subsequent funding for specific projects. However, some bridge funding was required from the academic department once the initial funding ran out. Analyses of the data have resulted so far in one funded grant, over a dozen publications, and a great deal of pilot data, "spinoff" projects, and, as in the Dutch experience, a treasure trove for students and fellows.

Because of the potential importance of this kind of research, state and local governments and private 
foundations may be potential sources of funding for such projects in the United States. Collaborative relationships with such organizations should be explored and developed. Advocacy at a national level will also be important.

\section{Summary}

Longitudinal research is essential to develop family practice, and despite its inherent difficulties, there are ways to move forward and overcome the barriers. There is no magic button to press though; longitudinal data are collected by toiling researchers, trying to create the most optimal conditions. Examples of success and learning from the experiences of others, essential tools in research development, are the best way forward.

\section{Box: Learning Points of Workshop}

- Continuity of care requires longitudinal research.

- PBRNs are well-positioned to support longitudinal research.

- Longitudinal research can be either concurrent or nonconcurrent (prospective, retrospective, or mixed).

- Recruitment and retention in longitudinal studies conducted in family practice can be high because of the relationships between patients and their personal physicians.

- Standardization of classifications and definitions, training of staff, and documentation of changes in practice across time are necessary to insure consistency of data over time.

- Informed consent is an important issue especially because views about the ethical conduct of research changes over time.

- Statistical analyses of longitudinal data can be quite complex, so statistical advice is recommended during the planning phases of the project.

- Whereas traditional funding sources are often reluctant to fund longitudinal studies, such studies do not have to be inordinately expensive, nontraditional sources may be available, and the long-term payoffs for an academic department may be worth the investment.

\section{References}

1. Wonca Europe. The European Definition of General Practice/Family Medicine (accessed August 8,
2005). Available from http://www.Wonca Europe 2002.

2. Martin CJ, Avant RF, Bowman MA, et al. The future of family medicine: a collaborative project of the family medicine community. Ann Fam Med 2004;2 Suppl 1:S3-32.

3. van Weel C. Longitudinal research and data collection in primary care. Ann Fam Med 2005;3 Suppl $1: \mathrm{S} 46-51$.

4. van Weel-Baumgarten $E$, van den Bosch $W$, van den Hoogen H, Zitman FG. Ten year follow-up of depression after diagnosis in general practice. Br J Gen Pract 1998;48:1643-6.

5. van Weel C, Smith H, Beasley JW. Family practice research networks. Experience from three countries. J Fam Pract 2000;49:938-43.

6. van Weel-Baumgarten EM, van den Bosch WJ, van den Hoogen HJ, Zitman FG. The validity of the diagnosis of depression in general practice: is using criteria for diagnosis as a routine the answer? Br J Gen Pract 2000;50:284-7.

7. van Weel-Baumgarten EM, van den Bosch WHJM, van den Hoogen HJM, Zitman FG. The long-term perspective: a study of psychopathology and health status of patients with a history of depression, more than 15 years after the first episode. Gen Hosp Psychiatry 2000;22:399-404.

8. Mold JW, Vesely SK, Keyl BA, Scenk JB, Roberts $M$. The prevalence, predictors, and consequences of peripheral sensory neuropathy in older patients. J Am Board Fam Pract 2004;17:309-18.

9. van Weel-Baumgarten EM, van den Bosch WJHM, Hekster YA, van den Hoogen HJ, Zitman FG. Treatment of depression related to recurrence: 10 year follow-up in general practice. J Clin Pharm Therapeutics 2000;25:61-6.

10. Diggle PJ, Liang KY, Zeger SL. Analysis of longitudinal data. New York: Oxford University Press; 1994.

11. Fairclough DL. Design and analysis of quality of life studies in clinical trials. Boca Raton (FL): Chapman \& Hall/CRC; 2002.

12. Singer JD, Willett JB. Applied longitudinal data analysis. New York: Oxford University Press; 2003.

13. Donner A, Birkett N, Buck C. Randomization by cluster-sample size requirements and analysis. Am J Epidemiol 1981;114:906-14.

14. Wears RL. Advanced statistics. Acad Emerg Med 2002;9:330-41.

15. Killip S, Mabfoud Z, Pearce K. What is an intracluster correlation coefficient? Crucial concepts for primary care researchers. Ann Fam Med 2004;2:204-8.

16. Reed JF. Adjusted chi-square statistics: application to clustered binary data in primary care. Ann Fam Med 2004;2:201-3. 